\title{
Properties of Turbulence in the Reconnection Exhaust: Numerical Simulations Compared with Observations
}

\author{
F. Pucci $^{1}$, S. Servidio ${ }^{2}$, L. Sorriso-Valvo ${ }^{3}$, V. Olshevsky ${ }^{1}$, W. H. Matthaeus ${ }^{4}$, F. Malara ${ }^{2}$, \\ M. V. Goldman ${ }^{5}$, D. L. Newman ${ }^{5}$, and G. Lapenta ${ }^{1}$ \\ ${ }^{1}$ Center for Mathematical Plasma Astrophysics, Department Wiskunde, KU Leuven, 200B Celestijnenlaan, Leuven, B-3001, Belgium; francesco.pucci@kuleuven.be \\ ${ }^{2}$ Dipartimento di Fisica, Università della Calabria, I-87036 Cosenza, Italy \\ ${ }^{3}$ Nanotec-CNR, U.O.S. di Cosenza, Via P. Bucci, Cubo 31C, Arcavacata di Rende, I-87036, Italy \\ ${ }^{4}$ Department of Physics and Astronomy, University of Delaware, 217 Sharp Lab, Newark, DE 19716, USA \\ ${ }^{5}$ University of Colorado, Boulder, CO 80309, USA \\ Received 2017 January 16; revised 2017 April 4; accepted 2017 April 26; published 2017 May 24
}

\begin{abstract}
The properties of the turbulence that develops in the outflows of magnetic reconnection have been investigated using self-consistent plasma simulations, in three dimensions. As commonly observed in space plasmas, magnetic reconnection is characterized by the presence of turbulence. Here we provide a direct comparison of our simulations with reported observations of reconnection events in the magnetotail, investigating the properties of the electromagnetic field and the energy conversion mechanisms. In particular, simulations show the development of a turbulent cascade consistent with spacecraft observations, statistics of the dissipation mechanisms in the turbulent outflows similar to the ones observed in reconnection jets in the magnetotail, and that the properties of turbulence vary as a function of the distance from the reconnecting X-line.
\end{abstract}

Key words: magnetic reconnection - methods: numerical - turbulence

\section{Introduction}

Turbulence and magnetic reconnection are two fundamental phenomena in space plasmas. The former is responsible for the cascade of magnetic and ordered kinetic energy from large scales, where the energy is injected, to small scales, where the energy can be transformed to particle heating and acceleration. The latter consists in the reconfiguration of magnetic field topology with the effect of decreasing magnetic energy in favor of particle heating or acceleration. These two phenomena are not separate in nature; on the contrary, they often go "hand-inhand" (Matthaeus \& Velli 2011). The effects of turbulence on magnetic reconnection have been widely studied in magnetohydrodynamics (MHD; Matthaeus \& Lamkin 1986; Lazarian \& Vishniac 1999; Loureiro et al. 2007, 2009; Kowal et al. 2012), while remaining not well understood in the context of collisionless plasmas. It has also been shown that the generation of strong small-scale current sheets in the turbulent cascade provides the conditions for the onset of reconnection, making the latter a fundamental ingredient of the former (Servidio et al. 2009, 2011).

On the other hand, the self-generation of turbulence in magnetic reconnection has been studied as well theoretically in 2D (Matthaeus \& Lamkin 1986; Malara et al. 1991, 1992; Lapenta 2008; Bhattacharjee et al. 2009) and 3D (Beresnyak 2016; Huang \& Bhattacharjee 2016) numerical simulations of MHD. In recent years, thanks to the steady increase of the available computational resources, the full kinetic self-consistent description of 3D reconnection has become a reality. It has been shown that in 3D new phenomena arise that change the picture of how and where the magnetic energy is converted to plasma energy (Daughton et al. 2011; Lapenta et al. 2015). Many of these discoveries concern the physics of the outflows of reconnection: Vapirev et al. (2013) showed that an interchange instability develops at the interface between the plasma ejected from the first reconnection site and the ambient plasma, Lapenta et al. (2015) showed that in the reconnection outflows a large number of secondary reconnection sites develop, and Leonardis et al. (2013) showed that intermittent turbulence develops in the outflows.

Observations reveal the presence of a large number of reconnection events, from large to small scales (Retinò et al. 2007; Greco et al. 2016). Analogously, it is commonly observed that large-scale exhausts are far from being in a laminar and regular regime, showing instead the clear manifestation of turbulence (Osman et al. 2015). More specifically, spacecraft observations of reconnection have also revealed the presence of turbulence within the ion diffusion region (Eastwood et al. 2009). In the inertial subrange, electric and magnetic fluctuations both followed a classical $k^{-5 / 3}$ power law; at higher frequencies, the spectral indices were near -1 and $-8 / 3$, respectively.

In this paper, motivated by spacecraft observations, such as the studies by Eastwood et al. (2009) and Osman et al. (2015), we study the properties of turbulence in the outflows of reconnection by means of a 3D kinetic numerical simulation. We recover many features of turbulence that develop in the reconnection outflows as observed in the Earth magnetotail. We show that a turbulent energy spectrum develops at kinetic scale as a consequence of reconnection. The slopes of the electric and magnetic energy spectra at ion scales are found to be consistent with observations, along with the scale at which the two spectra depart from each other. We better characterize where and how the energy exchange between fields and particles happens in a reconnection event. Our results show that dissipation takes place mainly in the outflows and is intermittent. Moreover, we show how the properties of the turbulence vary moving away from the X-point in the outflow direction. Our results are relevant to the physics of the magnetotail and could be useful in better understanding the ongoing Magnetospheric Multiscale (MMS) mission observation of that region. 


\section{Numerical Simulation and Analysis}

In our simulation, we consider a plasma made of protons and electrons. The initial configuration is the classical Harris equilibrium:

$$
\boldsymbol{B}=B_{0 x} \tanh (y / \delta) \boldsymbol{e}_{x}+B_{0 z} \boldsymbol{e}_{z}, n=n_{0 b}+\frac{n_{0}}{\cosh ^{2}(y / \delta)} .
$$

The coordinates are chosen as follows: $x$ along the sheared component of the magnetic field (Earth-Sun direction in the Earth magnetotail), $y$ in the direction of the gradients (northsouth in the magnetotail), and $z$ along the current and the guide field (dawn-dusk in the magnetotail). For both species, the particle distribution function is Maxwellian with spatially homogeneous temperature. A uniform background $n_{0 b}$ is added in the form of a nondrifting Maxwellian at the same temperature of the main Harris plasma. We solve the Vlasov-Maxwell equations for the two species using the semi-implicit Particle In Cell code iPIC3D (Brackbill \& Forslund 1982; Markidis et al. 2010; Lapenta 2012). We consider a $3 \mathrm{D}$ box of shape $[40,15,10] d_{p}$, where $d_{p}$ is the proton inertial length, which is resolved by a Cartesian grid of $[720,270,228]$ cells, each one initially populated with 125 particles. We use a realistic mass ratio, $m_{p} / m_{e}=1836$, which fixes the spatial resolution to $\Delta x=d_{p} / 18 \sim 2 d_{e}$, where $d_{e}$ is the electron inertial length. The proton and electron thermal speeds are $0.0063 c$ and $0.12 c$, respectively, at the initial time, where $c$ is the speed of light, resulting in a temperature ratio of $T_{p} / T_{e}=5$. The thickness of the initial current sheet is set to $\delta=0.5 d_{p}$, the density of the background such that $n_{0 b} / n_{0}=0.1$, and the case of small guide field is considered: $B_{0 z} / B_{0 x}=0.1$. We impose open boundary conditions in the $x$ and $y$ direction and periodicity along $z$. The magnetic reconnection process is initialized with a perturbation of the $z$ component of the vector potential localized in the center of the domain (Vapirev et al. 2013). The plasma ejected from the first reconnection sites encounters the ambient plasma and piles up, forming the reconnection front. This front is unstable, producing magnetic fluctuations and initiating the turbulent cascade. It moves toward the boundaries and eventually exits the simulation box. We stop the simulation when the reconnection front has already started moving and is far enough from both the boundaries to study the turbulence that develops in front of it. This time corresponds to $t=23.3 \Omega_{c p^{-1}}$, where $\Omega_{c p}$ is the proton cyclotron frequency computed using the asymptotic magnetic field, which is the time at which we performed the bulk of our analysis.

\subsection{Electric and Magnetic Spectra}

In order to establish a first connection between plasma simulations of turbulent reconnection and the observations, we computed the power spectral densities of the fluctuations. Because of the inhomogeneous background, it is important to first establish the anisotropy level and, in general, the 3D properties of turbulence. As we are interested in the fluctuations produced by magnetic reconnection, we define the magnetic fluctuations as

$$
\boldsymbol{b}(\boldsymbol{x})=\boldsymbol{B}(\boldsymbol{x})-\left\langle B_{x}(\boldsymbol{x})\right\rangle_{x, z} \boldsymbol{e}_{x}-\langle\boldsymbol{B}(\boldsymbol{x})\rangle_{x, y, z},
$$

where $\langle\bullet\rangle$ represents spatial averaging in the (two or three) directions indicated by the suffix. The above definition subtracts both mean fields and the large-scale shear: the second term on the right-hand side represents the signature of the background Harris sheet that is still present at the time we are analyzing, while the last term is the (small) guide field. The reduced autocorrelation function, computed in each direction, averaging over the entire volume, is defined as $C\left(r_{j}\right)=\left\langle\boldsymbol{b}\left(\boldsymbol{x}+r_{j} \hat{\boldsymbol{r}}_{j}\right) \cdot \boldsymbol{b}(\boldsymbol{x})\right\rangle_{x, y, z} / b^{2}$. Here $j=x, y, z, \hat{\boldsymbol{r}}_{j}$ are the unit vectors in the three directions, and $b^{2}$ is the average magnetic energy of the fluctuations, i.e., $b^{2}=C(0)$.

In an infinite size system, for regular statistics, the autocorrelation function tends to zero for large values of the displacements, indicating convergence of the moments. We computed $C\left(r_{j}\right)$ at the peak of the nonlinear activity, measuring the correlation length of the fluctuations $\lambda_{C_{j}}$ as the displacement at which the correlation function is reduced by a factor $1 / e$. Using this $e$-folding procedure, we measured $\lambda_{C_{x}}=$ $4.4 d_{p}, \lambda_{C_{y}}=2.8 d_{p}$, and $\lambda_{C_{z}}=1.7 d_{p}$. This difference between correlation lengths indicates spectral (or correlation) anisotropy among the three main directions. The large-scale (energycontaining) vortexes are more elongated in the $x$ direction (along the reconnecting field). There is a secondary anisotropy due to the presence of the shear along $y$, which suggests that the coherent structures have the shortest extension along the periodic direction $z$. This observation is therefore in agreement with the anisotropic geometry of the problem.

In order to compute the power spectra, we used Hanningwindowing in the $x$ and $y$ directions, isolating the central exhausts. The window size and sharpness have been varied, verifying that the chosen parameters do not alter the spectrum significantly. The 3D energy spectra of the magnetic field confirm what was found about the correlation lengths. It is worth noting, however, that the main anisotropy direction is along $x$ and that the anisotropy in the $y z$ plane is smaller and negligible at scales $r \ll \lambda_{C_{z}}$. This effect of isotropization is typical of small-scale turbulence. In our case, isotropy is recovered in the $\left(k_{y}, k_{z}\right)$ plane for $k_{y z} d_{p}>1.5$, with $k_{y z}=\sqrt{k_{y}^{2}+k_{z}^{2}}$. Therefore, it is reasonable to compute the total energy spectrum by integrating over $k_{x}$ and computing concentric isotropic shells in the $\left(k_{y}, k_{z}\right)$ plane. The results for the electric and magnetic energy are shown in Figure 1. In agreement with space plasma observations (Bale et al. 2005; Eastwood et al. 2009), the two spectra exhibit a different power-law decay at proton scales, with the electric spectrum proceeding at subproton scales with spectral index $\sim-1$, while the magnetic spectrum behaves more like $\sim k^{-8 / 3}$. The characteristic spatial scale at which the two spectra depart is $k d_{p} \sim 1$, in accordance with observations. In order to compare the two spectral indices, the electric field power spectrum has been rescaled by a factor of $5 \times 10^{3}$. Eastwood et al. (2009) found a factor of $\sim 9 \times 10^{4}$, an order of magnitude larger. However, in our simulation the Alfvén speed and the ion thermal speed are $\sim 1.5$ and $\sim 3.0$ times bigger, respectively, than their typical values in observation. From a dimensional analysis of Faraday's law we get that $E / B \approx v$, where $E, B$, and $v$ are characteristic quantities. Hence, it is expected that the electric activity will increase if the typical plasma velocities increase. As stated in the original work about the electric/ magnetic departure (Bale et al. 2005), the explanation of this phenomenon is still an open issue. The spectral behavior can be interpreted within two main approaches. The first involves the 


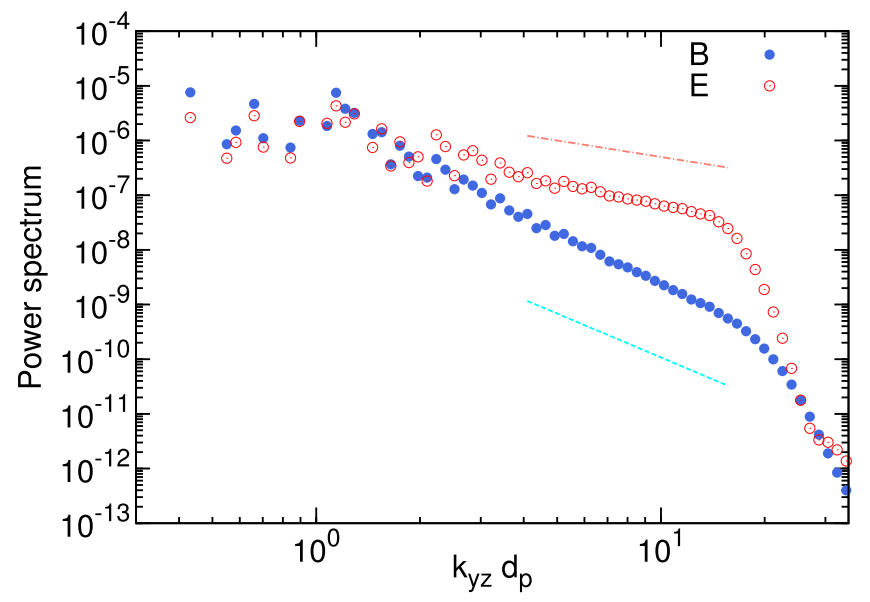

Figure 1. Power spectra of magnetic (blue bullets) and electric (open red circles) fields as a function of the perpendicular $k$-vector (with respect to the reconnecting field direction). Spectra have been reduced along $k_{x}$. The dotteddashed red line and dashed blue line indicate the behavior of the observations for the magnetic $\left(\propto k^{-8 / 3}\right)$ and electric spectra $\left(\propto k^{-1}\right)$, respectively.

presence of kinetic Alfvén waves, which might be favored in this configuration since the $y-z$ direction is oblique to the mean field (about $85^{\circ}$; Gary \& Nishimura 2004). If one assumes a turbulent cascade of KAW-like fluctuations, decorrelating on a timescale comparable to the linear KAW period (Howes et al. 2008), scaling arguments predict that the magnetic energy spectrum behaves like $k^{-7 / 3}$ while the electric-energy spectrum flattens to $k^{-1 / 3}$ (both different from the spectra observed here). However, the strong shear here can alter and decorrelate these waves, favoring the other explanation. The second interpretation is more qualitative and follows simple dimensional arguments. At scales smaller than the ion skin depth, the electric field is dominated by the Hall term and, as described in Matteini et al. (2017), the electric fluctuation behaves like

$$
\frac{\delta E}{V_{A}} \sim\left(k d_{p}\right) \delta B,
$$

where $V_{A}$ is the characteristic Alfvén speed. If fluctuations manifest an inertial range, then $P_{E}(k) \sim k^{\alpha_{E}}$ and $P_{B}(k) \sim k^{\alpha_{B}}$. From the above relation one gets simply that $\alpha_{E}=\alpha_{B}+2$. This relation is qualitatively observed in our case, as can be seen from Figure 1. It is also worth remarking how the above interpretation still holds in such an anisotropic and inhomogeneous system, where spectra need to be carefully extracted removing large-scale background profiles and border effects.

\subsection{Energy Exchange between Fields and Particles}

The energy exchange between fields and particles is governed by the term $\boldsymbol{J} \cdot \boldsymbol{E}$, where $\boldsymbol{J}$ is the total current sum of proton and electron contributions and $\boldsymbol{E}$ is the electric field. When $D_{l}=\boldsymbol{J} \cdot \boldsymbol{E}$ is positive, the energy is flowing from the fields to the particles; when it is negative, energy is passing from particles to fields. It is sometimes referred to as the dissipative term, even though the energy transfer from the magnetic field to particles is not always an irreversible process, and so it does not strictly imply dissipation. Despite this fact, for the purpose of our paper we will keep this definition, also used elsewhere (Zenitani et al. 2011; Wan et al. 2012; Olshevsky et al. 2015, 2016), and from now on we will use $D_{l}$ as a proxy for dissipation or more properly energy release
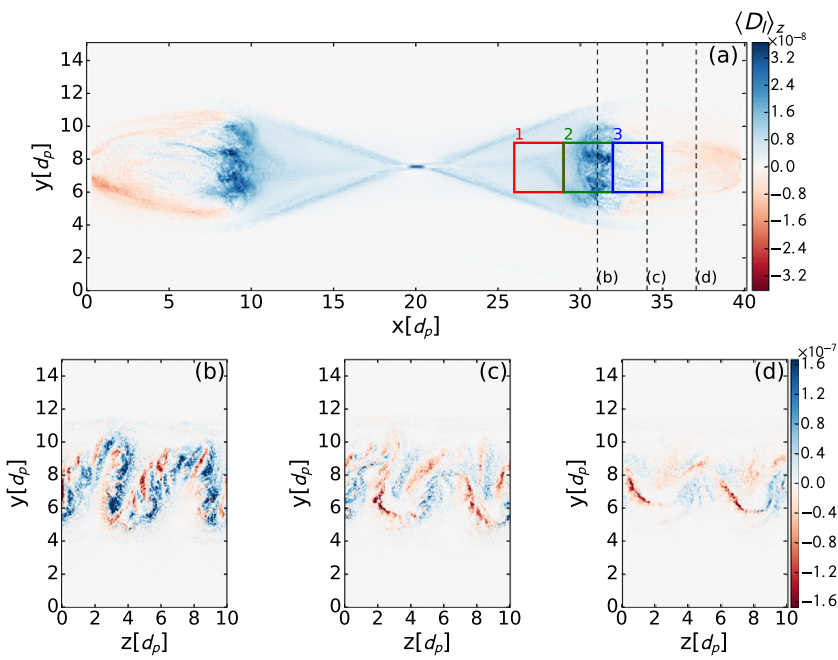

Figure 2. Energy exchange $D_{l}=\boldsymbol{J} \cdot \boldsymbol{E}$ (a) in the $x y$ plane averaged in the $z$ direction, and in the $y z$ plane at (b) $x=31 d_{p}$, (c) $x=34 d_{p}$, and (d) $x=37 d_{p}$. The $x$-line is located at $x=20 d_{p}$. The three boxes in panel (a) are the ones used for the statistical analysis presented in Section 2.2.

from the electromagnetic field (in the laboratory frame). A 2D plot of $D_{l}$ integrated in the $z$ direction is shown in Figure 2. Note that this differs from the similarly motivated dissipation surrogate used previously in turbulence studies, e.g., Wan et al. (2012), which computed maps of $\boldsymbol{J} \cdot \boldsymbol{E}$ in the electron fluid frame (Zenitani et al. 2011). As shown in other works, in collisionless magnetic reconnection $D_{l}$ is not concentrated only around the first reconnection site (Lapenta et al. 2014, 2015). In fact, it takes nonzero values in a wider region contained in the outflows (panel (a)). Moreover, $D_{l}$ is strongly inhomogeneous inside the outflows. In order to characterize this inhomogeneity, we plotted $D_{l}$ in the plane facing the outflows, $y z$, at three different positions along $x: 31 d_{p}, 34 d_{p}$, and $37 d_{p}$. The largest values of $D_{l}$ are found in the region where the plasma ejected by reconnection encounters the ambient plasma and is decelerated, near $x=31 d_{p}$ in the right outflow. $D_{l}$ is stronger in that position and decreases moving outward from the first reconnection site in the outflow direction. Note that $D_{l}$ has in general both positive and negative values, but in the considered region its average is always positive, indicating a net flow of energy from fields to particles. As has been noticed in previous studies conducted in the contest of MHD (TanDokoro \& Fujimoto 2005; Guo et al. 2014) or by means of a kinetic model (Vapirev et al. 2013), the region of interface between the low-density plasma ejected by magnetic reconnection and the high-density ambient plasma is unstable. Guo et al. (2014) have interpreted that such instability has an interchange instability where the deceleration existing between lighter and denser plasma plays a role that is equivalent to gravity in the Rayleigh-Taylor instability. Through a 3D MHD model of magnetic reconnection the authors of this study related the phenomenology of this instability to supra-arcade downflows observed in active region coronae above post-eruption flare arcades. It is worth noticing that, even though our study is meant to apply to Earth's magnetotail, the value of beta considered in the present work, $\beta \sim 0.8$ in the asymptotic region, is comparable to $\beta=0.5$ in Guo et al. (2014). For this reason it is reasonable to compare our findings obtained using a kinetic model to what was found for MHD. We observe a similar phenomenology to what was found by Guo et al. 

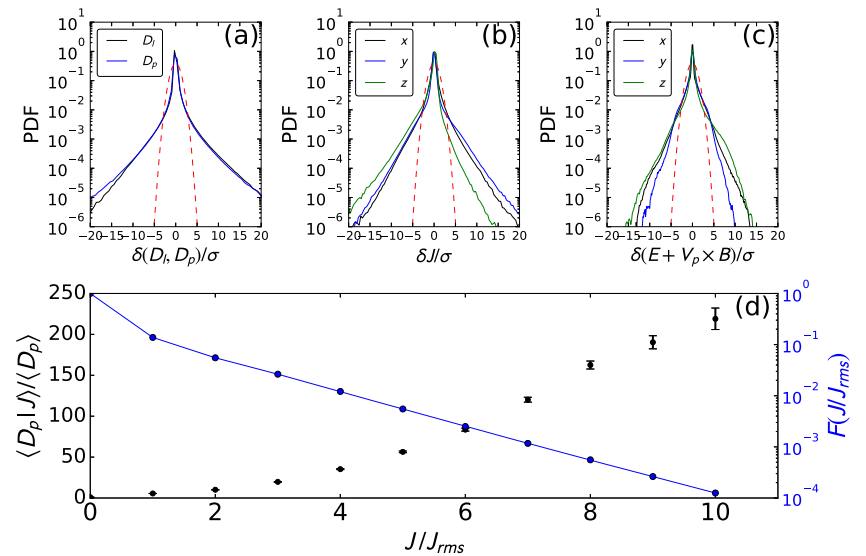

Figure 3. PDFs of (a) $D_{l}$ and $D_{p}$, (b) $\boldsymbol{J}$, and (c) $\boldsymbol{E}_{\boldsymbol{p}}$. Red dashed lines represent the normalized Gaussian curve. (d) Mean $D_{p}$ conditioned on local current density thresholds and (right axis) fraction $F$ of the full box data used to compute the averages.

(2014), i.e., finger-like structures of low-density plasma that penetrate the high-density plasma in the reconnection outflow. These structures, which extend in the $x$ direction, are visible in Figure 2(b), where a 2D plot of the dissipation proxy in the $y z$ plane at $x=31$ is presented. We notice that $D_{l}$ has two evident modulations, one at large scales with a period of around $L_{z} / 2$, and one at smaller scales that overlaps the large-scale one. The former is due to the kink of the equilibrium current sheet (Ricci et al. 2004; Yin et al. 2008); the latter is due to the aforementioned interchange instability. Our results show that the width of the filaments that are formed by the instability is of the order of $0.5 d_{p}$ (and so is much larger than $d_{e}$ ).

In order to give a better description of how energy is converted and to compare our results with observations, we performed a statistical analysis similar to what was presented by Osman et al. (2015), where a dissipation analysis was performed on observational data in a magnetic reconnection outflow in the magnetotail. It is worth noticing that in Osman et al. (2015) the statistics were made from temporal data collected by the satellites crossing the reconnection outflow, while in our case the whole simulation box is used as a single sample. In panel (a) of Figure 3 the probability density functions (PDFs) of $\delta D_{l}=D_{l}-\left\langle D_{l}\right\rangle_{x, y, z}$ and $\delta D_{p}=D_{p}-\left\langle D_{p}\right\rangle_{x, y, z}$ are plotted. $D_{p}$ represents the dissipation proxy in the proton reference frame and is given by $D_{p}=\boldsymbol{J} \cdot \boldsymbol{E}_{p}$, where $\boldsymbol{E}_{p}=\left(\boldsymbol{E}+\boldsymbol{V}_{p} \times \boldsymbol{B}\right)$ is the electric field in the proton reference frame, with $\boldsymbol{V}_{p}$ representing the proton bulk velocity. The two PDFs are compared with the normalized Gaussian distribution (plotted as a dashed red line). They strongly depart from Gaussian distributions, presenting instead high tails up to several standard deviations $\sigma$. In panels (b) and (c), we present separately the PDFs of the two terms that compose $D_{p}$. In agreement with what was found in the space measurement, the PDFs of $\boldsymbol{J}$ and $\boldsymbol{E}_{p}$ are both non-Gaussian. In panel (d), the average $D_{p}$ conditioned to a threshold current density is shown. The plot is constructed as follows: a threshold in the current density magnitude is considered and the average of $D_{p}$ is computed using all those points in the domain where the value of the current is bigger than the fixed threshold. This average is then normalized to the average of $D_{p}$ on all points, which gives by definition $\left\langle D_{p} \mid J=0\right\rangle /\left\langle D_{p}\right\rangle=1$. The black points in the plots represent the result of such computation for different values of the threshold. The blue curve represents the filling factors, i.e., the fraction of points used for computing the average with respect to the total number of points in the sample. The average of $D_{p}$ strongly increases when higher thresholds are considered up to $J / J_{\mathrm{rms}}=10$. Currents sheets are present that have values bigger than $10 J_{\text {rms }}$, but for those values the statistics is too poor to draw reliable conclusions.

The comparison of our results with the observations by Osman et al. (2015) is striking. The PDFs of dissipation proxy $D_{l}$ and $D_{p}$ are not just qualitatively similar to what was found in observations, but within the limit of observational resolution they agree also in a quantitative way. We show here that the non-Gaussian tail of dissipation proxy PDFs could extend up to values that are 10 times or more higher than their typical standard deviation. A similar conclusion can be drawn for what concerns the conditioned average of $D_{p}$. We show that current peaks are present that are 10 times bigger than the typical value of the current, where the dissipation proxy $D_{p}$ takes values that are more than 100 times bigger than the average $D_{p}$. A qualitatively similar result was found in the observations, but with less intense current density and, as a consequence, values of the dissipation proxy where observed. It is worth remarking that in the paper by Osman et al. (2015) current density was computed using the curlometer technique. This technique uses magnetic field measures taken at the same time from different satellites and computes the curl of the magnetic field knowing the satellite's position at that time. Thus, the curlometer estimation of current density becomes more imprecise as far as intense current gradients at scales around the satellite separation are concerned. This can result in neglecting strong gradients at small scales. This limitation is overcome in our simulation, where the current is directly computed as a fluid moment of the particle distribution functions. Our results confirm that the exchange of energy is local, with larger values of $D_{p}$ localized in very small volume filling structures. This evidence and the presence of non-Gaussian PDFs of dissipation proxies suggest that magnetic reconnection produces smallscale current sheets that are sites of strong events of energy exchange between fields and particles. Concisely stated, all these statistics indicate that dissipation in a reconnection event is intermittent. A similar conclusion was reached by Wan et al. (2012), who examined the electron frame dissipation surrogate conditioned on magnitude of current density.

The above statistical analysis was performed considering the whole simulation box, providing information about the average properties of the dissipation proxy in the reconnection events. In order to obtain a more detailed description, and to identify in which place the intermittent energy exchange actually occurs, we perform the previous statistical analysis in three different regions of the simulation, by selecting three boxes located in the right reconnection outflow at three different distances from the $\mathrm{X}$-point. The boxes are identified by $6<y / d_{p}<9$ (reconnection zone), $0<z / d_{p}<10$ (full domain in $z$ ), and with $\mathrm{BOX}_{1}=\left\{26<x / d_{p}<29\right\}, \quad \mathrm{BOX}_{2}=\left\{29<x / d_{p}<32\right\}$, $\mathrm{BOX}_{3}=\left\{32<x / d_{p}<35\right\}$ (see Figure 2). Moving further in the direction of the outflows, the PDFs become non-Gaussian. This transition happens between $\mathrm{BOX}_{1}$ and $\mathrm{BOX}_{2}$ (Figure 4, panels (a)-(c)). Moreover, passing from $\mathrm{BOX}_{2}$ to $\mathrm{BOX}_{3}$, the PDFs of $D_{p}$ and $D_{l}$ become more similar, suggesting that proton inertia becomes less important in the exhaust of reconnection. The evolution of the conditioned average is interesting as well (Figure 4, panels (b), (d), and (f)). Moving from $\mathrm{BOX}_{1}$ to $\mathrm{BOX}_{3}$, the conditioned average of $D_{p}$ increases for bigger values of the current density threshold. This is more evident in the direct 

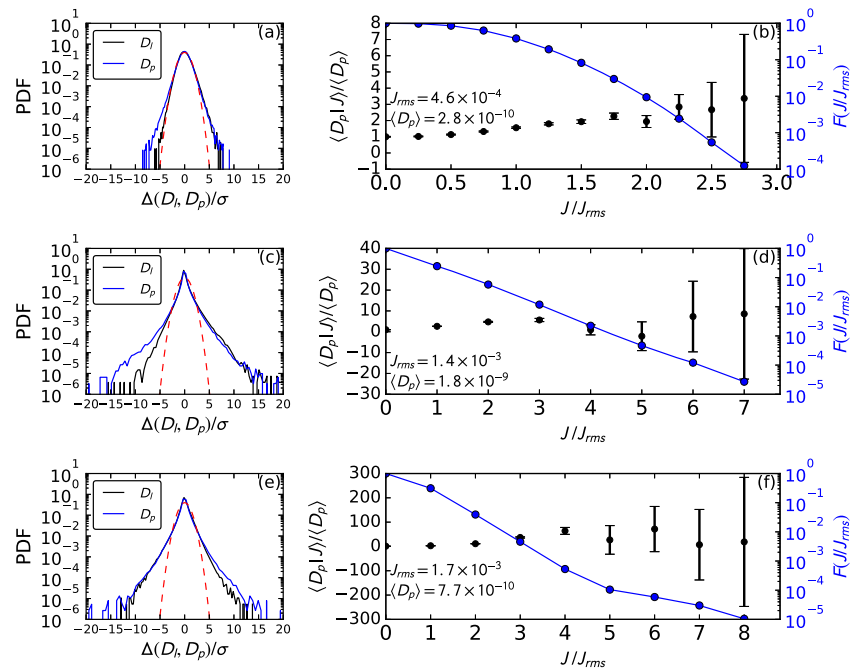

Figure 4. PDFs of $D_{l}$ and $D_{p}$ in (a) $\mathrm{BOX}_{1}$, (c) $\mathrm{BOX}_{2}$, and (e) $\mathrm{BOX}_{3}$. Conditioned average of $D_{p}$ and filling factors $F$ in (b) $\mathrm{BOX}_{1}$, (d) $\mathrm{BOX}_{2}$, and (f) $\mathrm{BOX}_{3}$.

comparison shown in Figure 5, where the normalized conditioned average grows from $\mathrm{BOX}_{1}$ to $\mathrm{BOX}_{3}$ (panel (a)). Similarly, the structure's filling factor shows higher tails passing from $\mathrm{BOX}_{1}$ to $\mathrm{BOX}_{2}$ and $\mathrm{BOX}_{3}$. The intermittent nature of energy exchange is recovered for $\mathrm{BOX}_{2}$ and $\mathrm{BOX}_{3}$, which are at the level of the interface between the plasma ejected from reconnection and the ambient plasma and farther away from the $\mathrm{X}$-point, respectively. In order to exclude the possibility that such a result depended on the particular time considered, we performed the same analysis following the motion of the interface (analysis not shown here). The time analysis confirmed that the statistical behavior of the dissipation proxy, which indicates intermittency, is found starting from the reconnection front toward the outflow and moving away from the X-point. This means that the instability previously discussed plays an important role in dissipation in the reconnection outflows and needs to be further investigated.

\section{Discussion and Conclusions}

We have used a 3D kinetic numerical simulation to study the properties of the turbulence that develops in the outflow of magnetic reconnection using parameters typical of the Earth magnetotail with realistic mass ratio. The reader might be concerned that the run shown here, while it has a realistic mass ratio, is somewhat lacking in spatial resolution at electron scales. To address this issue, we carried out another run with unrealistic mass ratio $(m p / m e=256)$ but four times better resolution of electron scales. We found minor differences, mainly in the sharpness of features at small scales, but all of the main conclusions shown here were adequately confirmed. Simulations have shown that turbulence in the outflows is anisotropic, with large scales dominated by fluctuations whose wavevector is directed in the direction of the reconnecting magnetic field. Magnetic and electric turbulent energy spectra follow two different power laws at scales smaller than the proton inertial length, with slopes that are in agreement with observations.

Like the turbulent activity, the energy exchange between fields and particles is concentrated in the outflows, where the
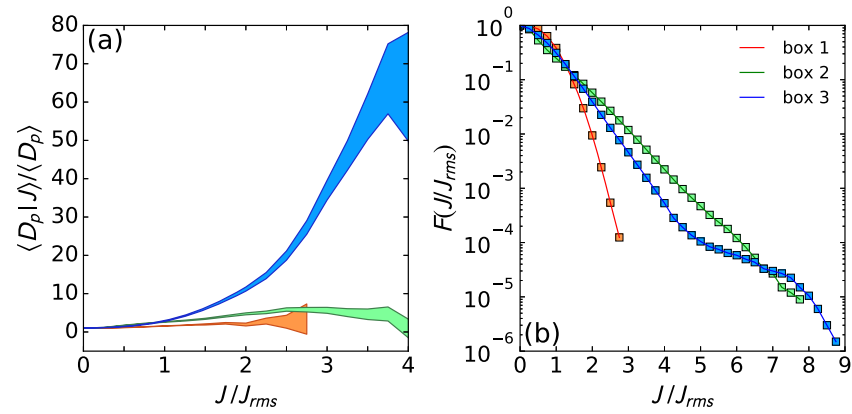

Figure 5. (a) Conditioned average of $D_{p}$ and (b) filling factor in BOX 1 (red), BOX 2 (green), and BOX 3 (blue).

strongest values of dissipation are found at the interface between the plasma ejected by reconnection and the ambient plasma. This region is unstable to a Rayleigh-Taylor-type instability, which has also been observed in MHD simulations of supra-arcade downflows in solar coronae. We showed the presence of such instability in our simulations and provided an estimation of the width of the finger-like structures in plasma density that form because of the instability. We found a typical width of $0.5 d_{p}$, a result that could not have been obtained in MHD studies, which by definition cannot resolve proton scales.

Statistical analysis of the dissipation proxy confirms that the energy exchange between fields and particles occurs in a small volume filling structure where the value of the current is much stronger than its rms. Our results match well to observations and show that a more intense intermittent event could be found if a more precise measure of the current density were available. In fact, the current sheets produced by the turbulent activity compared to their rms values are stronger in numerical simulation compared to observations. This difference could be due to the curlometer technique used to estimate current density from magnetic field measurements. Currents sheets are found in simulations that are more intense than 10 times their typical values, but their statistics is too poor to assess any reasonable conclusion. There is an indication that it is possible for locations where very intense current sheets are present to be sites of negative values of $D_{p}$, meaning passage of energy from particles to fields at small scales, but the nature of such an effect has to be studied more in depth with better-resolved runs, which are left for a future work.

Finally, we showed that the properties of the turbulence produced in the outflows vary in space, becoming intermittent when the reconnection front is crossed moving far from the $\mathrm{X}$-point. The reconnection front is also the site of the aforementioned instability, which seems to play a key role in the development of turbulence in the outflow, the study of which is left for a future work as well.

We believe that these results could be used to better explain the upcoming $M M S$ mission observations of the magnetotail.

The present work was supported by NASA MMS grant nos. NNX08AO84G and NNX14AC39G, by the NASA Heliophysics Grand Challenge grant NNX14AI63G, by the Onderzoekfonds KU Leuven (Research Fund KU Leuven), by the Interuniversity Attraction Poles Programme of the Belgian Science Policy Office (IAP P7/08 CHARM), and by the DEEP-ER project of the European Commission. The simulations were conducted on NASA (NAS and NCCS) supercomputers, on the VSC Flemish 
supercomputing center, and on the facilities provided by PRACE research infrastructure Tier- 0 grants. This research used resources of the National Energy Research Scientific Computing Center, a DOE Office of the Science User Facility supported by the Office of Science of the U.S. Department of Energy under contract No. DE-AC02-05CH11231.

\section{References}

Bale, S., Kellogg, P., Mozer, F., Horbury, T., \& Reme, H. 2005, PhRvL, 94, 215002

Beresnyak, A. 2016, ApJ, 834, 47

Bhattacharjee, A., Huang, Y.-M., Yang, H., \& Rogers, B. 2009, PhPl, 16, 112102

Brackbill, J., \& Forslund, D. 1982, JCoPh, 46, 271

Daughton, W., Roytershteyn, V., Karimabadi, H., et al. 2011, NatPh, 7, 539

Eastwood, J., Phan, T., Bale, S., \& Tjulin, A. 2009, PhRvL, 102, 035001

Gary, S. P., \& Nishimura, K. 2004, JGRA, 109, A02109

Greco, A., Perri, S., Servidio, S., Yordanova, E., \& Veltri, P. 2016, ApJL, 823, L39

Guo, L.-J., Huang, Y.-M., Bhattacharjee, A., \& Innes, D. E. 2014, ApJL, 796, L29

Howes, G., Dorland, W., Cowley, S., et al. 2008, PhRvL, 100, 065004

Huang, Y.-M., \& Bhattacharjee, A. 2016, ApJ, 818, 20

Kowal, G., Lazarian, A., Vishniac, E. T., \& Otmianowska-Mazur, K. 2012, NPGeo, 19, 297

Lapenta, G. 2008, PhRvL, 100, 235001

Lapenta, G. 2012, JCoPh, 231, 795

Lapenta, G., Goldman, M., Newman, D., Markidis, S., \& Divin, A. 2014, PhPl, 21, 055702
Lapenta, G., Markidis, S., Goldman, M. V., \& Newman, D. L. 2015, NatPh, 11,690

Lazarian, A., \& Vishniac, E. T. 1999, ApJ, 517, 700

Leonardis, E., Chapman, S. C., Daughton, W., Roytershteyn, V., \& Karimabadi, H. 2013, PhRvL, 110, 205002

Loureiro, N., Schekochihin, A., \& Cowley, S. 2007, PhPl, 14, 100703

Loureiro, N., Uzdensky, D., Schekochihin, A., Cowley, S., \& Yousef, T. 2009, MNRAS, 399, L146

Malara, F., Veltri, P., \& Carbone, V. 1991, PhFlB, 3, 1801

Malara, F., Veltri, P., \& Carbone, V. 1992, PhFlB, 4, 3070

Markidis, S., Lapenta, G., Rizwan-uddin, et al. 2010, Math. Comp. Simul., 80, 1509

Matteini, L., Alexandrova, O., Chen, C. H. K., \& Lacombe, C. 2017, MNRAS, 466, 945

Matthaeus, W., \& Lamkin, S. L. 1986, PhFl, 29, 2513

Matthaeus, W., \& Velli, M. 2011, SSRv, 160, 145

Olshevsky, V., Deca, J., Divin, A., et al. 2016, ApJ, 819, 52

Olshevsky, V., Lapenta, G., Markidis, S., \& Divin, A. 2015, JPIPh, 81, 325810105

Osman, K., Kiyani, K., Matthaeus, W., et al. 2015, ApJL, 815, L24

Retinò, A., Sundkvist, D., Vaivads, A., et al. 2007, NatPh, 3, 236

Ricci, P., Lapenta, G., \& Brackbill, J. 2004, GeoRL, 31, L06801

Servidio, S., Dmitruk, P., Greco, A., et al. 2011, NPGeo, 18, 675

Servidio, S., Matthaeus, W., Shay, M., Cassak, P., \& Dmitruk, P. 2009, PhRvL, 102, 115003

TanDokoro, R., \& Fujimoto, M. 2005, GeoRL, 32, L23102

Vapirev, A., Lapenta, G., Divin, A., et al. 2013, JGRA, 118, 1435

Wan, M., Matthaeus, W., Karimabadi, H., et al. 2012, PhRvL, 109, 195001

Yin, L., Daughton, W., Karimabadi, H., et al. 2008, PhRvL, 101, 125001

Zenitani, S., Hesse, M., Klimas, A., \& Kuznetsova, M. 2011, PhRvL, 106, 195003 